\title{
Monte Carlo Method-Based Behavioral Reliability Analysis of Fully-Mechanized Coal Mining Operators in Underground Noise Environment
}

\author{
Yuansheng WANG, Guoxun JING*, Shaoshuai GUO, Fei ZHOU
}

\begin{abstract}
Sound is an important aspect in working environment, and underground strong noise environment imposes a serious impact on operators' physical and mental health and easily leads to people's unsafe behaviors, thus giving rise to accidents. How to quantitatively study operators' behavioral reliability is a study hotspot. To mitigate the noise impact on operators and reduce the occurrence rate of accidents due to human factors, the relational models between noisy working environment and human physiological indexes were established first through a laboratory simulation, and then a reliability integral model was obtained using performance function and limit state equation. Second, the established reliability integral model was numerically simulated based on Monte Carlo numerical simulation method to obtain its numerical solution, and operators' behavioral reliability values under different sound pressure levels (SPLs) on fully-mechanized coal mining face were calculated. The results show that the behavioral reliability of fully-mechanized coal mining operators is high with low accident occurrence rate under noise SPL of 50-70 dB. Under 70-90 dB, their behavioral reliability is 0.7092 with potential accident risks. The behavioral reliability is low when SPL is $90-110 \mathrm{~dB}$, under which accidents may easily take place. This study manifests that operators' behavioral reliability analysis under underground noise environment based on Monte Carlo method is of certain feasibility. The conclusions have a certain guiding significance for relieving human physical and mental harms incurred by noise, improving human behavioral reliability, reducing human errors and guaranteeing safety production.
\end{abstract}

\section{Keywords: behavioral reliability; fully-mechanized coal mining face; Monte Carlo method; noise; physiological index}

\section{INTRODUCTION}

Coal mining belongs to an industry with frequent safety accidents, and there are many factors influencing operators under the complex fully-mechanized coal mining environment. With the continuous improvement of coal mining technology, fully-mechanized coal mining is the main development direction of coal mining technology [1]. Sound is an important aspect in working environment, but some unnecessary sounds may become noises which harm workers' physical and psychological health [2]. Especially with the development of modern industry and scientific \& technological progress, more and more high-power and high-rotation-speed mechanical equipment has been applied to coal mine production, the noise pollution in coal mines is more serious, and the noise impact on fullymechanized coal mining face is especially serious [3-4]. According to statistical data of the State Administration of Work safety, a total of 190 among coal mine safety accidents during 15 years (2003-2018) occurred in fullymechanized coal mining faces, causing 760 deaths. Although an overall declining tendency was presented, the accidents due to human errors like illegal command, operation against rules, operation at risk, negligence, improper measures and misoperation accounted for $88.4 \%$ of total number of accidents occurring in fully-mechanized coal mining faces [5]. Human is a weak link in coal mine production. It is found by analyzing safety accidents due to human factors that there are two causes for safety accidents triggered by human errors: operators' psychological factors and human physiological factors [6-7]. Therefore, investigating the effect of noise on physiological and psychological indexes so as to establish operators' behavioral reliability model will be of great realistic significance.

However, most of the existing studies regarding coal mine safety have started from gas and dust explosion, and many studies have explored code of conduct for operators from the perspective of accidents due to human factors. Little attention has been paid to underground humanenvironment combining face in coal mine from the angle of systems engineering, and the effects of harsh underground working environment on operators' safety behaviors and behavioral reliability are ignored. Therefore, to solve the abovementioned research deficiencies, the effects of underground noise environment in coal mine on operators' behavioral reliability were comprehensively analyzed in this study from the angle of humanenvironment system, expecting to provide a certain reference for reducing human errors and improving operators' behavioral reliability.

\section{STATE OF THE ART}

Special underground noise pollution can be formed in coal mines due to numerous mechanical equipment, narrow working space, strong directivity of ventilation and large equipment load, etc. [8]. Underground strong noise will not only influence normal voice communication [9] but also exert quite complex impact on human. Among studies regarding the noise impact upon personnel, most of the existing literature has analyzed operators' psychology and behavior from medical angle, e.g. strong noise can accelerate operators' heart rate, cause various diseases like central nervous system dysfunction $[11,12]$, form severe physiological and psychological harms to miners $[13,14]$, and moreover, noise may easily divert miners' attention [15], lead to fatigue, severely influence miners' safety behavioral reliability, thus increasing the probability of accidence occurrence [16]. As for evaluation of impact of underground environment on personnel reliability, the correlation between historical data and influence factors is mostly analyzed through mathematical statistics method, the action degree of various factors on personnel reliability is determined, but there is lack of analysis from the angle of human-environment relationship. For instance, Zhang et al. used full-size analog machine from nuclear power plant to obtain basic parameters of characteristic HRA/HCR model and Qinshan nuclear power plant system through the operators' reliability test [17]. He et al. compared seven common HRA analysis methods and put forward 12 criteria for comparing these HRA methods [18]. Bertolini used fuzzy cognitive map (FCM) to investigate influence factors of human reliability and their relationship [19]. By 
investigating accidents due to human factors in coal mines, Chen et al. proposed a prediction and evaluation method for operator errors in coal mines and evaluated their operational reliability [20]. Wang and Ma analyzed the nonlinear dynamic process of human errors based on radial basis function neural network and applied it to reliability analysis of hoisting operators [21]. Zhang et al. quantitatively analyzed the reliability of deck officers under different control modes through the transformed triangle white function network [22]. Groth introduced hierarchical pragmatic influence factors (PIFs) which could be applied to qualitative and quantitative analysis of HRA, and improved the hierarchical setup of PIFs [23]. Wang et al. carried out a quantitative study on operators' behavioral reliability in transformer substation via cognitive reliability and error analysis model [24]. Chen et al. used CREAM method to quantitatively analyze back brake operators' reliability in one transformer substation by changing environmental factors [25]. Wang et al. combined growth theory and grey relation theory to quantify the control modes in cognitive reliability and error analysis methods, established HEP and grey relational degree function models and applied to human factor reliability analysis in coal mine production [26]. Podofillini used Bayes method for probability prediction of human errors and believed that this method could further refine human factor reliability algorithms [27].

To sum up, from the entry point of studies, predecessors' studies on noise are mostly from the medical angle, namely prevention and treatment of occupational diseases, and the obtained results are pathogeneses of degradation or impairment of body functions under most circumstances, but no reasonable and effective solutions have been raised. Second, personnel reliability is analyzed mostly using mathematical models from the perspective of human behaviors, and it is quite difficult to explain the complex nonlinear relations and coupling actions between all kinds of influence factors of underground safety system of coal mines.

Therefore, to solve the deficiencies of the existing studies, the realistic features of human factor engineering and working environment of fully-mechanized coal mining face were combined to design relevant tests according to knowledge of safety engineering science, environmental science, physiology and behavior. Based on the relation models of noisy working environment with human heart rate and blood pressure, a reliability integral model was obtained using performance function and limit state equation. Monte Carlo numerical simulation method was utilized to conduct numerical simulation of the established reliability integral model, the numerical solution of which was obtained, so as to calculate operators' behavioral reliability values under different sound pressure levels (SPLs) of fully-mechanized coal mining face. This study will provide a certain reference for relieving physical and mental harms caused by noise to human, improving their behavioral reliability, reducing human errors and guaranteeing safety production.

The remainder of this study is organized as follows: Section 3 expounds the laboratory investigation on the influence relationships between noisy working environment and various human physiological indexes and establishes a reliability model based on Monte Carlo numerical simulation. Section 4 presents the modelling results and analysis. The final section summarizes relevant conclusions.

\section{METHODOLOGY}

\subsection{Laboratory Investigation on Influence Relationship between Noisy Working Environment And Human Physiological Indexes}

On the basis of previous studies, sensitive physiological indexes greatly influenced by noise were selected in this test for measurement, namely heart rate, systolic pressure and diastolic pressure.

\subsubsection{Test Environment}

The physiological index measurement test was conducted on the test platform established by HumanMachine Laboratory of Henan Polytechnic University in China. The test only performed analog control of noise environment without considering cross impacts of other environmental factors on human physiological indexes. To ensure the reliability of test data, environmental parameters of the environmental simulation platform such as temperature, humidity and illuminance were set constant in order to keep them consistent with or similar to those of underground fully-mechanized coal mining face serving as the test sample.

\subsubsection{Test Objects}

A total of 62 technically skilled miners were randomly selected as the subjects. All of them had junior high school degree or above, aging from 30 to 40 years old, 160-170 $\mathrm{cm}$ in height and $60-75 \mathrm{~kg}$ in weight with normal uncorrected or corrected visual acuity, normal color perception, rich operating experience and good physical health. They worked carefully and could make efforts to cooperate in the test without drinking experience. The test objects all came from a coal mine subordinate to a group in Henan Province, China, and they represented the current mainstream group occupied in fully-mechanized coal mining in coal mines.

\subsubsection{Test Devices and Materials}

Physiological data in this test were measured using 24 $\mathrm{h}$ dynamic cardiotachometer and $24 \mathrm{~h}$ ambulatory blood pressure meter, respectively. The noise stimulus data used in the test derived from recorded sound materials of Shenhuo fully-mechanized coal mining face in Shangqiu City, Henan Province, China (YLY2.8 mineral highfidelity voice recording pen was used), and the noise sources included MG300/700-WD AC traction coal cutter, SGZ800/750 twin middle chain conveyor (no-load operation) and hydraulic power unit. The decibel and exposure time of stimulus materials were controlled with high-fidelity stereo surround sound equipment and stopwatch, respectively, where the maximum playing decibel value of sound equipment could reach $130 \mathrm{~dB}$.

\subsubsection{Concrete Testing Process}

The impact of noise on operators' physiological parameters was simulated in this test. By reference to actual noise environment of fully-mechanized coal mining face, the noise level in the experimental group was divided 
into 5 levels: $70 \mathrm{~dB}, 80 \mathrm{~dB}, 90 \mathrm{~dB}, 100 \mathrm{~dB}$ and $110 \mathrm{~dB}$, and the noise level in the control group was background noise level $40 \pm 2 \mathrm{~dB}$ of the test platform.

(1) $30 \mathrm{~min}$ before the test began, environmental parameters of the test platform were adjusted by the laboratory staff, who would then explain the testing process and operation requirements to the subjects, and the subjects would get their test numbers and laboratory apparatus.

(2) After the subjects got familiar with the test environment and their physiological indexes became stable, initial physiological index values displayed on corresponding instruments were read, and the laboratory staff made relevant records.

(3) The test of the control group started. The subjects exercised according to the set jogging speed and time (6 $\mathrm{km} / \mathrm{s}$ and $60 \mathrm{~min}$ ). Energy consumption of the subjects was made consistent with their regular operating intensity as much as possible. Physiological parameter data were recorded every other $5 \mathrm{~min}$. After the exercise was finished, the subjects would take $5 \mathrm{~min}$ rest and the laboratory staff would immediately record their physiological parameters.

(4) Noise stimulus test of the experimental group was begun. Experimental noise samples were played by the staff in the operating room, and the subjects would exercise according to the same jogging time and speed as the control group. Instrument data were recorded every other $5 \mathrm{~min}$. The subjects would take 5 min rest after finishing all exercises, and the laboratory staff would immediately record data.

(5) The whole testing process lasted $65 \mathrm{~min}$. The experimental instruments on the experimental environmental platform were closed and the subjects took rest in the laboratory. Light music would be played in the laboratory for $30 \mathrm{~min}$ after each experiment, and purified water and snacks were provided to the subjects in order to mitigate negative impact of noise environment on them.

(6) Experiments (1) to (4) were repeated every day. To avoid the disturbance caused by noise adaptation, noise tests at all levels were separately implemented, noise test of only one level should be arranged every day, and the whole test lasted five days.

\subsubsection{Laboratory Data Processing}

Microsoft Excel office software was used to summarize human physiological index data under noise stimulus and SPSS 20.0 was employed for test data processing and analysis.

\subsection{Reliability Modelling Based on Monte Carlo Numerical Simulation}

Human reliability value under noise environment is calculated according to the calculation method as seen in Eq. (1).

$$
\begin{aligned}
p_{s}= & P\left(\bigcap_{i=1}^{n} Z_{i}>0\right) \\
& =\int \ldots \iint_{0}^{+\infty} f\left(Z_{1}, Z_{2}, \ldots, Z_{n}\right) d_{z_{1}} d_{z_{2}} d_{z_{3}}, \ldots, d_{z_{n}}
\end{aligned}
$$

where $f\left(Z_{1}, Z_{2}, \ldots, Z_{n}\right)$ is comprehensive probability density function of performance function. The performance function $Z_{i}=R_{i}-S_{i}(i=1,2, \ldots, n)$ has a direct relation with utility index, but it is difficult to measure operators' physiological indexes in a real-time way, so operational utility indexes of operation under noise environment will be solved according to environmental factors of fully-mechanized coal mining face based on the relation models between operators' physiological indexes and environmental factors of fully-mechanized coal mining face previously established in the laboratory investigation, where $Z_{i}(y)$ is the function of operational utility index $i$ about noise environmental indexes of fullymechanized coal mining face, and then the reliability calculation is transformed into integral model establishment for environmental factor data of fullymechanized coal mining face.

It is quite hard to obtain analytical solutions because of the reliability calculation formula belonging to multiple integration. Monte Carlo numerical simulation method is used to solve numerical solutions of integrals [28-30]. The first method used, simulation error and expected accuracy are set as $\varepsilon=0.05$ and $\delta=0.001$, respectively, so it is only necessary to set $N_{0}=20000$.

\section{RESULTS ANALYSIS AND DISCUSSION 4.1 Correlation Analysis between Noise SPL and Physiological Indexes}

According to the laboratory test results, the relationships between noise SPL and physiological indexes are obtained.

(1) Fitting of noise and systolic pressure and residual plot:

It can be seen from Fig. 1 that with the increase of SPL, systolic pressure firstly declines and then increases. As shown in the residual plot, residual errors are under random distribution around the null point, indicating good model fitting effect. The following quadratic function is obtained through regression analysis:

where $R^{2}$ is the percentage of the dependent variable change which can be explained by the fitted model. In the Tab. $1, R^{2}=0.879$, meaning that the fitted equation is capable of explaining $87.9 \%$ changes of dependent variables. According to the model coefficients in the table, the functional expression of the fitted curve between systolic pressure and noise SPL can be obtained as below:

$$
y_{1}=0.012 x^{2}-1.724 x+174.214
$$

Table 1 Parameter table of sound pressure level and systolic function model

\begin{tabular}{|c|c|c|c|c|c|c|}
\hline \multirow{2}{*}{ Equation } & \multicolumn{3}{|c|}{ Model summary } & \multicolumn{3}{c|}{ Model coefficient } \\
\cline { 2 - 7 } & $R^{2}$ & $F$ & Significance & Constant & $b_{1}$ & $b_{2}$ \\
\hline $\begin{array}{c}\text { Quadratic } \\
\text { model }\end{array}$ & $\begin{array}{c}0.87 \\
9\end{array}$ & 18.1 & 0.005 & 174.214 & -1.724 & 0.012 \\
\hline
\end{tabular}

The model is significant above $95 \%$ confidence level, and $87.9 \%$ of actual data can be determined through the model.

(2) Fitting of noise and diastolic pressure and residual plot: 
It can be seen from Fig. 2 that diastolic pressure continuously increases with SPL. As observed from the residual plot, residual errors present random distribution around the null point, manifesting good model fitting effect. The following linear function is obtained based on a regression analysis:
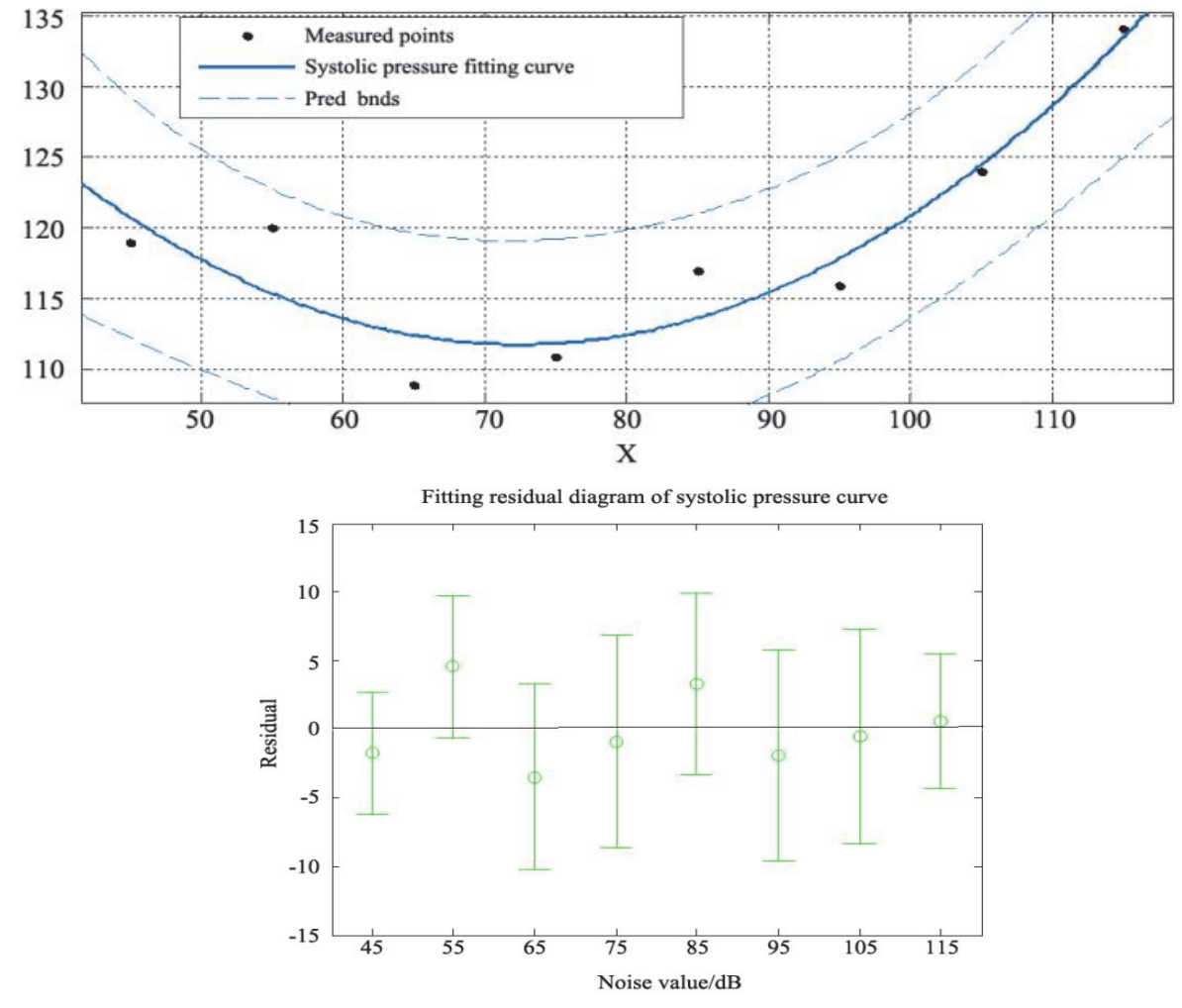

Figure 1 Fitting and residual chart of noise and systolic pressure
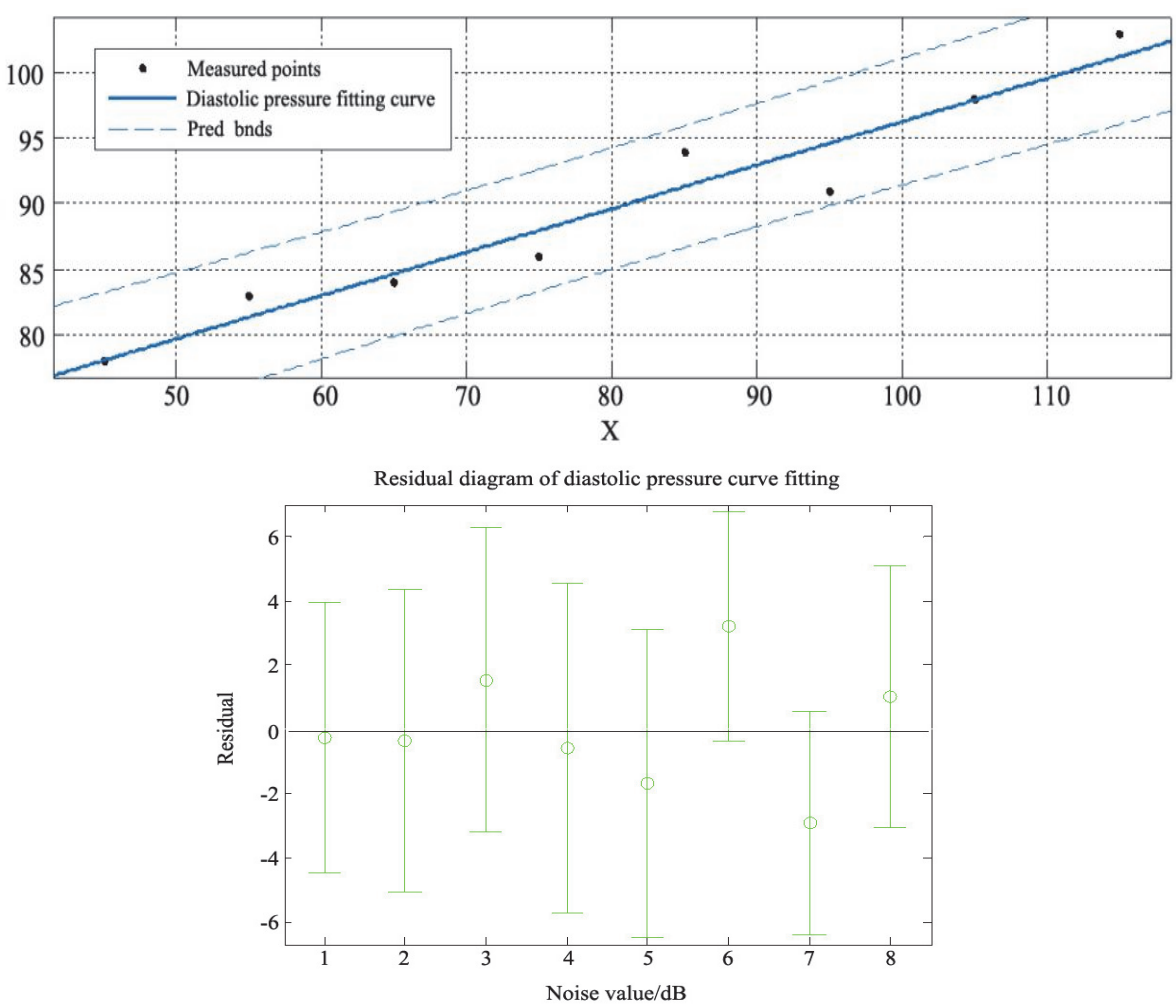

Figure 2 Fitting and residual plots of noise and diastolic blood pressure

In Tab. $2, R^{2}$ denotes the percentage of the dependent variable change which can be explained by the fitted model. $R^{2}=0.941$ means that the fitted equation can explain $94.1 \%$ changes of dependent variables. It can be obtained from the model coefficients in the table that the functional expression of the fitted curve between diastolic pressure and noise SPL is:

$y_{2}=0.311 x+65.268$ 
The model is significant above $95 \%$ confidence level, and $94.1 \%$ of actual data can be determined through the model.

Table 2 Parameter table of sound pressure level and diastolic pressure function model

\begin{tabular}{|c|c|c|c|c|c|}
\hline \multirow{2}{*}{ Equation } & \multicolumn{3}{|c|}{ Model summary } & \multicolumn{2}{c|}{ Model coefficient } \\
\cline { 2 - 6 } & $R^{2}$ & $F$ & Significance & Constant & $b_{1}$ \\
\hline Linear model & 0.941 & 95.81 & $6.543 \mathrm{e}-05$ & 65.268 & 0.311 \\
\hline
\end{tabular}

(3) Fitting of noise and heart rate and residual plot:

As shown in Fig. 3, heart rate presents a continuously rising tendency with the increase of SPL. It can be seen from the residual plot that residual errors are randomly distributed around the null point, manifesting good model fitting effect. The following quadratic function is obtained through the regression analysis:
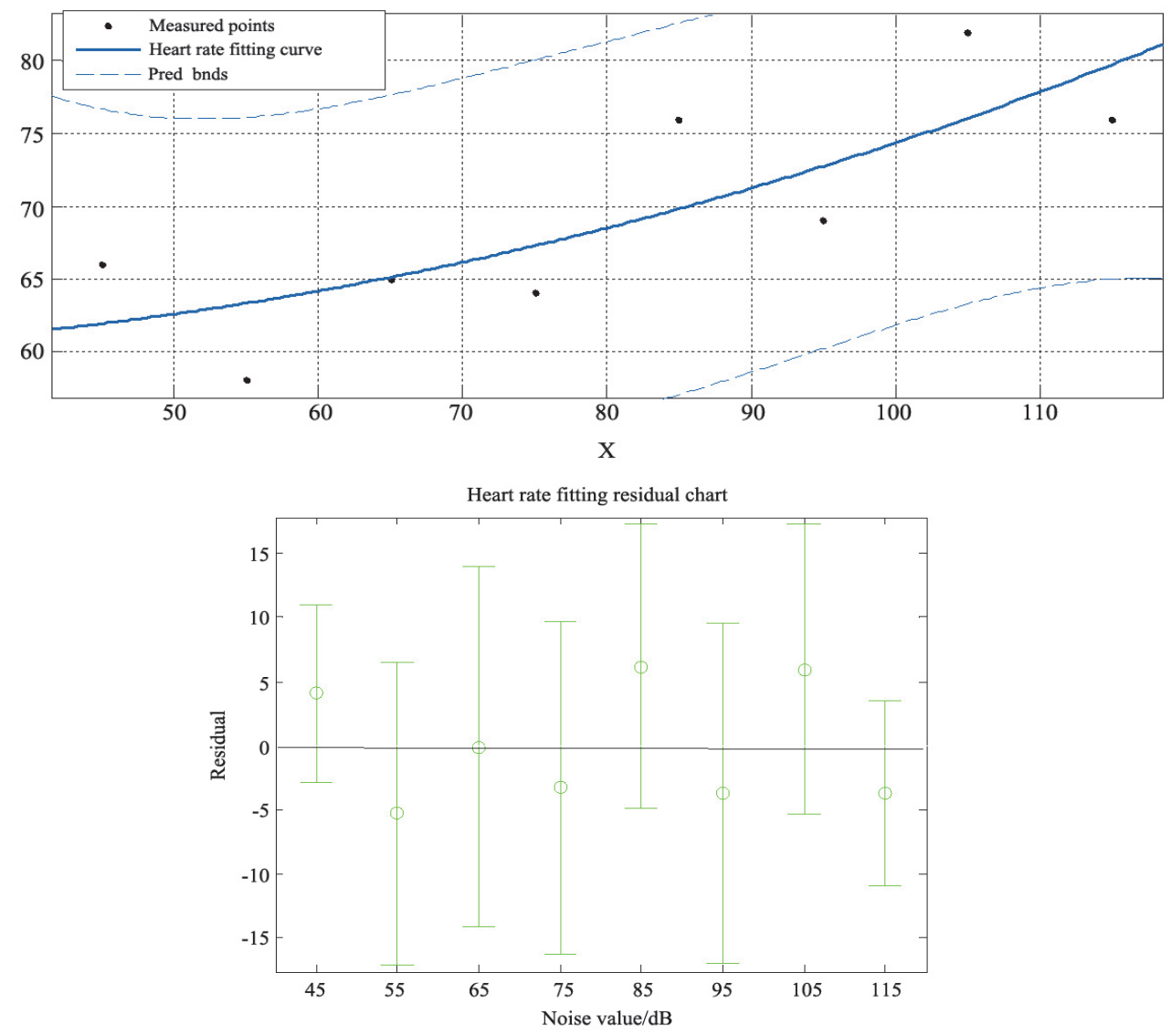

Figure 3 Fitting of noise and heart rate and residual plots

In Tab. $3, R^{2}$ expresses the percentage of the dependent variable change which can be explained by the fitted model. $R^{2}=0.941$ indicates that the fitted equation can explain $94.1 \%$ changes of dependent variables. It can be obtained from the model coefficients in the table that the functional expression of the fitted curve between heart rate and noise SPL is:

$$
y_{3}=0.008 x^{2}-1.023 x+96.787
$$

The model is significant above $95 \%$ confidence level, and $77.4 \%$ of actual data can be determined using the model.

Table 3 Sound pressure level and parameter table of heart rate function model

\begin{tabular}{|c|c|c|c|c|c|c|}
\hline \multirow{2}{*}{ Equation } & \multicolumn{3}{|c|}{ Model summary } & \multicolumn{3}{|c|}{ Model coefficient } \\
\cline { 2 - 7 } & $R^{2}$ & $F$ & Significance & Constant & $b_{1}$ & $b_{2}$ \\
\hline $\begin{array}{c}\text { Quadratic } \\
\text { model }\end{array}$ & 0.774 & 8.55 & 0.02 & 96.787 & -1.023 & 0.008 \\
\hline
\end{tabular}

Following a comprehensive analysis of Eqs. (2), (3) and (4), the relation model between noise and different factors is presented as below:

$$
\begin{aligned}
& Y=A \cdot X \\
& y=\left(y_{1}, y_{2}, y_{3}\right)^{\mathrm{T}}, x=\left(x^{2}, x, 1\right)^{\mathrm{T}},
\end{aligned}
$$

where $y=\left(y_{1}, y_{2}, y_{3}\right)^{\mathrm{T}}$ and $x=\left(x^{2}, x, 1\right)^{\mathrm{T}}$.

$$
\boldsymbol{A}=\left[\begin{array}{ccc}
0.012 & -.724 & 174.214 \\
0 & 0.311 & 65.268 \\
0.008 & -1.023 & 96.787
\end{array}\right]
$$

\subsection{Analysis of Reliability Simulation Results}

Random number of noise $x$ is generated using Monte Carlo method and substituted into formulas to calculate utility indexes: systolic pressure $y_{1}$, diastolic pressure $y_{2}$ and heart rate $y_{3}$, limit values $(140 \mathrm{mmHg}, 90 \mathrm{mmHg}$ and $95 \mathrm{bpm}$, respectively) of which are substituted to calculate their performance functions, and then overall performance function $Z$ and reliability $P f$ of operators are obtained (Tab. 4).

Table 4 Reliability result

\begin{tabular}{|c|c|c|c|}
\hline Sound pressure level / dB & $50-70$ & $70-90$ & $90-110$ \\
\hline Reliability & 1 & 0.7092 & 0 \\
\hline
\end{tabular}


The following results can be obtained by analyzing the above Tab. 4:

From the angle of operating environment, the reliability within $50-70 \mathrm{~dB}$ is 1 , indicating that human behaviors are reliable within this SPL. The reliability within $90-110 \mathrm{~dB}$ is 0 , so human behaviors are unreliable within this SPL. The reliability within $70-90 \mathrm{~dB}$ is 0.7092 , manifesting that human behaviors are of potential hazards within this SPL. Therefore, the reliability values are the maximum, medium and lowest within 50-70 dB, 70-90 dB and 90-110 dB, respectively.

\section{CONCLUSIONS}

Aiming at the impact of underground strong noise environment on operators and combining realistic features of human factor engineering and fully-mechanized coal mining face, relevant tests were designed according to knowledge in safety engineering science, environmental science, physiology and behavior. Operators' behavioral reliability model was constructed using Monte Carlo method to quantitatively investigate their behavioral reliability. The following conclusions were drawn:

(1) Operators' behavioral reliability was calculated based on Monte Carlo method, and a reliability calculation method was proposed for operators in underground noise environment of coal mines. Operators' behavioral reliability values within different SPLs were obtained. Monte Carlo method could quantify operators' behaviors in noise environment so as to better control adverse impact caused by noise to operators.

(2) According to the reliability calculation results through Monte Carlo method, the impact of noise on operators' behaviors varied with SPL. When SPL was smaller than $70 \mathrm{~dB}$, the behavioral reliability of fullymechanized coal mining workers was high, so accidents could hardly occur; Under $70 \mathrm{~dB}-90 \mathrm{~dB}$ condition, potential accident risks existed, and corresponding protective measures should be taken for operators. When SPL was higher than $90 \mathrm{~dB}$, operators' behavioral reliability was low, accidents might easily take place, so protective measures must be taken. The conclusions will be a certain guiding significance for relieving human physical and mental harms incurred by noise, improving human behavioral reliability, reducing human errors and guaranteeing safety production.

The study result proposed in this study is a behavioral reliability model of fully-mechanized coal mining workers in underground noise environment based on Monte Carlo method. Different from previous qualitative studies, this study can provide an accurate theoretical support for exploring the noise impact on operators' behaviors, but the influence of complex underground environment on operators' behavioral reliability remains to be further investigated.

\section{Acknowledgements}

The study was supported by National Natural Science Foundation of China (51774120, U1904210, 51674102, 51874121), key scientific and technological project of Henan Province (182102310002) and special fund support for basic scientific research fees of colleges and Universities (180104).

\section{REFERENCES}

[1] Zhang, J. S., Fu, J., Hao, H. Y. et al. (2020). Root causes of coal mine accidents: Characteristics of safety culture deficiencies based on accident statistics. Process Safety and Environmental Protection, (136), 78-91. https://doi.org/10.1016/j.psep.2020.01.024

[2] Georgiev, G., Balabanova, I., Kogias, P. et al. (2018). Identification of sine, squire, triangle and sawtooth waveforms with uniform white and inverse $\mathrm{F}$ noises by adaptive neuro: Fuzzy interface system. Journal of Engineering Scienceand Technology Review, 11(3), 128-132. https://doi.org/10.25103/jestr.113.17

[3] Tang, Z. P., Chen, Z. X., Sun, J. P. et al. (2019). Noise prediction of traction gear in high-speed electric multiple unit. International Journal of Simulation Modelling, 18(4), 720-731. https://doi.org/10.2507//JSIMM18(4)CO20

[4] Zeng, J., Yao, Q. G., Zhang, Y. S. et al. (2019). Optimal path selection for emergency relief supplies after mine disasters. International Journal of Simulation Modelling, 18(3), 476487. https://doi.org/10.2507/IJSIMM18(3)486

[5] Zhang, J., Xu, K., You, G. et al. (2019). Causation analysis of risk coupling of gas explosion accident in Chinese underground coal mines. Risk Analysis, 39(7), 1634-1646. https://doi.org/10.1111/risa.13311

[6] Liu, R. L., Cheng, W. M., Yu, Y. B. et al. (2018). Human factors analysis of major coal mine accidents in China based on the HFACS-CM model and AHP method. International Journal of Industrial Ergonomics, 68, 270-279. https://doi.org/10.1016/j.ergon

[7] Marinescu, A. C., Sharples, S., Ritchie, A. C. et al. (2018). Physiological Parameter Response to Variation of Mental Workload. Human Factors, 60(1), 31-56. https://doi.org/10.1177/0018720817733101

[8] Zhao, X., Niu, C. C., Wang, J. et al. (2019).Thermodynamic model of steam injection pipeline considering the effect of time and phase change. Journal of Power Technologies, 99(02), 123-130.

[9] Lu, J., Wang, Y., \& Chen, J. (2018).Detection of tectonically deformed coal using model-based joint inversion of multicomponent seismic data. Energies, 11, 829-838. https://doi.org/10.3390/en11040829

[10] Erceg, A. (2019). Information security: threat from employees. Tehnički Glasnik, 13(2), 123-128. https://doi.org/10.31803/tg-20180717222848

[11] Xia, X., Chen, Z. G., \& Wei, W. (2018). Research on monitoring and prewarning system of accident in the coal mine based on big data. Scientific Programming, 9308742. https://doi.org/10.1155/2018/9308742

[12] Korchut, A. \& Korchut, W. (2017). The relationship between psychomotor efficiency and selected personality traits of people exposed to noise and vibration stimuli. Procedia Engineering, 199, 200-205. https://doi.org/10.1016/j.proeng.2017.09.274

[13] Bramhall, N., Beach, E., Epp, B. et al. (2019). The search for noise-induced cochlear synaptopathy in humans: Mission impossible. Hearing Research, 6(377), 88-103. https://doi.org/10.1016/j.heares.2019.02.016

[14] Votinov, M. (2020). The main directions of the humanization of industrial objects in urban environment. Tehnički Glasnik, 14(01), 60-65. https://doi.org/10.31803/tg-20190213110424

[15] Takacs, B. C., Guffey, S. E., Wu, M. Y. et al. (2015). Comparison of noise reduction values for fit tests and work in coal mines. Journal of the Acoustical Society of America, 137(4), 2377-2379. https://doi.org/10.1121/1.4920638 
[16] Zhang, Z. Y., Zhang, Z., \& Yang, J. F. (2018). Risk Factors of Secondary Accidents Based on the Bayesian Hierarchical Model. Journal of Engineering Science \& Technology Review, 11(5), 53-60. https://doi.org/10.25103/jestr.115.07

[17] Zhang, L., Huang, X. R., Zhao, B. Q. et al. (2005). The operator reliability experiment at Qinshan nuclearpower plant by the simulator. Engineering Science, (2), 41-46. https://doi.org/CNKI:SUN:GCKX.0.2005-02-007

[18] He, X. H. \& Gao, J. (2005). Comparison of human reliability analysis methods. Nuclear Power Engineering, (06), 627630. https://doi.org/10.3969/j.issn.0258-0926.2005.06.021

[19] Bertolini, M. (2007). Assessment of human reliability factors:A fuzzy cognitive maps approach. International Journal of Industrial Ergonomics, 37(5), 405-413. https://doi.org/10.1016/j.ergon.2005.12.009

[20] Chen, J. \& Cao, Q. G. (2007). Prediction and evaluation of human error in coal production. Mining Safety and Environmental Protection, (1), 78-81. https://doi.org/CNKI:SUN:ENER.0.2007-01-031

[21] Wang, H. D. \& Ma, C. Z. (2012). Human reliability prediction of lifting operation based on RBF neural network. China Safety Science Journal, 22(7), 42-47. https://doi.org/10.16265/j.cnki.issn1003-3033.2012.07.008

[22] Zhang, J. P., Chen, W. J., Zhang, H. et al. (2012). CREAMbased reliability analysis on OOW avoiding danger at sea. China Safety Science Journal, 22(09), 90-96. https://doi.org/10.16265/j.cnki.issn1003-3033.2012.09.012

[23] Groth, K. M. \& Mosleh, A. (2012).A data-informed PIF hierarchy for model-based Human Reliability Analysis. Reliability Engineering \& System Safety, 108, 154-174. https://doi.org/10.1016/j.ress.2012.08.006

[24] Wang, W., Gu, W., \& Kang, X. P. (2013). A method of human reliability analysis in substation. Energy Engineering, (1), 10-13. https://doi.org/10.16189/j.cnki.nygc.2012.05.016

[25] Lu, H. B., Wang, M., Guo, C. X. et al. (2013). A quantitative method for human reliability in power system based on CREAM. Power System Protection and Control, 41(5), 3742. https://doi.org/CNKl:SUN:JDQW.0.2013-05-008

[26] Wang, D., Liu, L., \& He, H. B. (2013). Improvement and application of human reliability analysis methods in coal mining production. Journal of Inner Mongolia University (Philosophy and Social Sciences), 45(4), 60-65. https://doi.org/10.13484/j.cnki.ndxbzsb.2013.04.011

[27] Podofillini, L. \& Dang, V. N. (2013). A Bayesian approach to treat expert-elicited probabilities in human reliability analysis model construction. Reliability Engineering \& System Safety, 117, 52-64. https://doi.org/10.1016/j.ress.2013.03.015

[28] Andreas, P. (2018). An adaptive longterm electricity price forecasting modelling using Monte Carlo simulation. Journal of Power Technologies, 98(3), 277-283.

[29] Janekova， J., Fabianova, J., \& Fabian, M. (2019). Assessment of Economic Efficiency and Risk of the Project Using Simulation. International Journal of Simulation Modelling, 18(2), 242-253. https://doi.org/10.2507/IJSIMM18(2)467

[30] Kosec, P., Skec, S., \& Miler, D. (2020). A comparison of the tolerance analysis methods in the open-loop assembly, Advances in Production Engineering \& Management, 15(1), 44-56. https://doi.org/10.14743/apem2020.1.348

\section{Contact information:}

Yuansheng WANG, PhD, Lecturer

School of Aviation, Anyang Institute of Technology,

Anyang, Henan, China

E-mail: 20190026@ayit.edu.cn

Guoxun JING, PhD, Professor

(Corresponding author)

School of Aviation, Anyang Institute of Technology,

Anyang, Henan, China

E-mail:guoxunj@163.com

Shaoshuai GUO, PhD candidate

School of Safety, Henan Polytechnic University,

Jiaozuo, China

E-mail: guoss6887@163.com

Fei ZHOU, PhD, Postdoctor

School of Safety, Henan Polytechnic University,

Jiaozuo, China

E-mail: zhoufeifei870511@163.com 